\title{
Joint cooperative relay scheme for spectrum- efficient usage and capacity improvement in cognitive radio networks
}

\author{
Qixun Zhang ${ }^{*}$, Zhiyong Feng and Ping Zhang
}

\begin{abstract}
In order to improve the efficiency of spectrum resource usage and the capacity of wireless networks, cooperative relay techniques which utilize the vacant spectrum of primary users for secondary users' data transmission have been applied in cognitive radio networks. Considering the dynamic time-varying vacant spectrum resources and achievable rate on different channels at relay nodes (RN), the traditional fixed time slot allocation scheme for cooperative RNs has the bottleneck for further improving the spectrum usage efficiency and system throughput. Therefore, the joint cooperative relay scheme with RN selection, channel allocation and dynamic time slot allocation (DyTSA), is designed to increase the spectrum usage efficiency and system capacity by dynamic tuning DyTSA ratio to adapt to the changing radio environment in multiple RNs serving multiple destinations scenario. Propositions of the proposed scheme are proved theoretically by closed-form solutions. Numerical results verify the effectiveness and correctness of the proposed scheme.
\end{abstract}

Keywords: cooperative relay, cognitive radio networks

\section{Introduction}

Based on measurement results unveiled by Federal Communications Commission (FCC) reports in [1,2], precious radio spectrum resources are underutilized and a large number of spectrum holes exist under traditional fixed spectrum assignment rules, which grant exclusive access to primary users (PU) and pay little attention to spectrum usage efficiency. Considering the changing radio environment and low spectrum usage efficiency, cognitive radio (CR) [3] technologies have been introduced with flexible spectrum assignment schemes to improve the spectrum usage efficiency. Furthermore, based on software-defined radio (SDR) [4] and CR [3] technologies, novel cognitive techniques with multi-domain radio environment cognition, autonomous decision making, self-reconfiguration, and intelligent learning abilities are proposed to improve both the spectrum usage efficiency and end-to-end (e2e)

\footnotetext{
* Correspondence: zqx830311@gmail.com

Wireless Technology Innovation Institute (WTI), Key Laboratory of Universal

Wireless Communications Ministry of Education, Information and

Telecommunication Engineering of Beijing University of Posts and

Telecommunications (BUPT), Haidian Dist. Xitucheng Rd. Beijing 100876, P.R.
} China

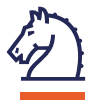

Springer

(C) 2012 Zhang et al; licensee Springer. This is an Open Access article distributed under the terms of the Creative Commons Attribution License (http://creativecommons.org/licenses/by/2.0), which permits unrestricted use, distribution, and reproduction in any medium, provided the original work is properly cited. network performance in cognitive radio networks (CRNs) [5].

However, challenges and problems on how to allocate spectrum to different secondary users (SU) with unbalanced spectrum resources and user demands in CRNs still exist which attract many attentions in recent research studies. By using vacant spectrum resources of $\mathrm{PU}$ for $\mathrm{SU}$ data transmission, cooperative relay technique, which utilizes the resource-rich nodes to serve the resource-starving nodes as a relay, has been considered as one of the key technologies to improve spectrum usage efficiency and enhance system throughput in CRNs. In the literature, many research works have been conceived on cooperative relay techniques in CRNs for spectrum efficiency enhancement. In [6], the cooperative spectrum sensing techniques are used to enhance the reliability of detecting PU in CRNs, and a cognitive space-time-frequency coding technique has been presented to adjust its coding structure by adapting itself to the dynamic spectrum environment. And the outage performance of relay-assisted cognitive wireless relay network is evaluated and quantified within the peak power constraints for spectrum sharing in [7]. Besides, the stable throughput techniques are designed in [8] by using 
SU as a relay for PU link, whose benefits depend on the network topology.

Furthermore, the distributed relay node $(\mathrm{RN})$ selection and routing scheme is proposed with better system coverage and spectrum efficiency compared to the centralized scheme in $[9,10]$. Based on buyer and seller game model, the distributed RN selection and power control algorithms have been designed in [11] to decrease the signalling cost in traditional centralized resource allocation scheme. Moreover, the joint RN assignment and fiow routing optimization scheme has been proposed by using novel components to speed-up computation time of branch-and-cut framework in multi-hop relay networks in [12]. Besides, the linear marking mechanism based optimal RN assignment scheme has been designed with formal proof of the linear complexity in [13]. Multihop relay routing strategies and the NNR and FNR strategies are proposed in [14] to enhance the spectrum usage and e2e system performance in a two-dimensional geometric network in Rayleigh fading channel. By introducing the pricing variables in OFDMA cellular system, a utility maximization framework has been proposed in [15] for joint RN selection, power and bandwidth allocation to optimize the physical-layer transmission strategies for user traffic demands. To maximize the throughput of relay network, the throughput optimal network control policy has been proposed in [16] to stabilize the network for any arrival rate in its stability region.

Due to equipment limitations in transceivers, RNs can not transmit and receive data on orthogonal channel at the same time for concurrent sessions. Thus, the halfduplex method by transmitting and receiving at different time slots for RNs is paid much attention for real implementation purposes. As described in $[17,18]$, a centralized heuristic solution has been proposed to address the relay selection and spectrum allocation problem under an infrastructure-based secondary network architecture in CRNs to improve spectrum efficiency. However, the constrains assumed by existing works in [18] that the transmission rate of each channel is identical and the time slot allocation scheme is fixed with half time to receive and the other half to transmit are not always applicable in terms of the time-varying channel condition in practical wireless network environment. Hence, how to achieve the high spectrum efficiency and system throughput under the condition of variant achievable rate on different channels and different user's demands with cooperative relay in CRNs is still an open issue.

Therefore, the dynamic time slot allocation (DyTSA) scheme is proposed in this article by dynamic tuning the time slots allocated on each relay link for receiving and transmitting, to improve spectrum efficiency and system capacity. The proposed DyTSA scheme considers the match up of variant achievable rate on different channels and user's demands. In multiple RNs serving multiple destinations (MR-MD) scenario, the DyTSA scheme is analyzed and proved thoroughly under different scenarios with closed-form solutions. Moreover, the joint RN selection, appropriate vacant channel allocation and DyTSA scheme is designed, which is also regarded as a crosslayer optimization solution. Numerical results with different SU density conditions verify the performance improvement on system capacity and spectrum efficiency in CRNs. The rest of the article is organized as follows. Section 2 describes the system scenario and assumptions. Section 3 focuses on problem formulation. Propositions and proofs are described in Section 4. Section 5 describes the joint RN selection, channel allocation and DyTSA scheme. Section 6 focuses on the analysis of simulation results. Finally, Section 7 conclude the article.

\section{System scenario and assumptions}

The centralized cooperative relay scenario is shown in Figure 1 with the secondary access point (SAP) serving each SU via a direct link in a cooperative manner. It is assumed that one destination node can be served by multiple RNs and each RN can also serve several different destination nodes at the same time. As proposed in [17], each SU can send or receive data on multiple channels simultaneously with one CR equipment, but it cannot send and receive data simultaneously. Figure 2 depicts the fiow of the transmission process and the time slot allocation solution in MR-MD scenario on different channels using graph theory [19], jointly considering both the channel allocation and the RN selection schemes.

\section{Problem formulation}

The CRN with relay links is denoted as a graph $G=(V$, $E) . V=\left\{v_{0}, v_{1}, \ldots, v_{N}\right\}$ is a set of $N+1$ nodes with $v_{0}$ as the SAP and $v_{i}(i \neq 0)$ as SU. $E=\left\{e_{i j}\right\}$ denotes the set of direct links between each pair of nodes, where $e_{i j}=1$ denotes that direct link between $v_{i}$ and $v_{j}$ exists, and 0 otherwise. It is assumed that the available spectrum resource is divided into $K$ channels with equal bandwidth $W$ and $A=\left\{a_{i}^{k}\right\}$ denotes the set of available channel at each node, where $a_{i}^{k}=1$ means that channel $k$ is available at $v_{i} . R=\left\{r_{i j}\right\}_{N \times N}$ denotes the set of relay relation between each pair of SUs except SAP, where $r_{i j}=1$ means that $v_{j}$ acts as a RN for $v_{i}$, and 0 otherwise. $X=\left\{x_{i j}^{k}\right\}$ denotes the set of channel allocation on each link, where $x_{i j}^{k}=1$ depicts that channel $k$ is allocated to link $e_{i j}$ for data transmission, and 0 otherwise. $C=\left\{c_{i j}^{k}\right\}$ denotes the set of achievable rate between $v_{i}$ and $v_{j}$ on channel $k$ with 


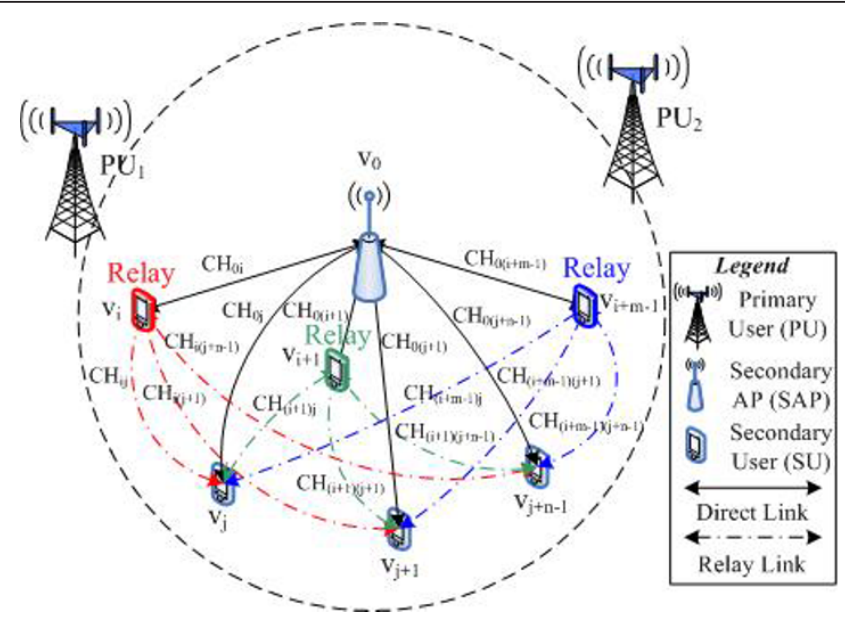

Figure 1 Scenario of DyTSA scheme in MR-MD scenario.

bandwidth $W$ and $c_{i j}^{k}\left(c_{i j}^{k} \geq 0\right)$ is calculated in (1). $H=\left\{h_{i j}^{k}\right\}$ denotes the channel-state of different channels on various links, where $h_{i j}^{k}$ means the channel-state information of channel $k$ on link $e_{i j}$, $P$ denotes the transmit power and $N_{0}$ as the background noise power.

$$
c_{i j}^{k}=W \log \left(1+\frac{\left|h_{i j}^{k}\right|^{2} \cdot P}{N_{0}}\right)
$$

There are two types of transmissions for cooperative relay in CRNs scenario: the direct transmission (from SAP to the destination node) and the relay transmission (from SAP to the destination node via the RN). Due to different channel conditions, the achievable rate on each link is not identical as assumed in [18] by $c$ and the fixed equal time slot allocation scheme is neither efficient nor applicable with different achievable rates on different channels in dynamic changing wireless network environment. Therefore, the DyTSA scheme, which allocates different length of time slots for receiving and transmitting at the $\mathrm{RN}$ in terms of variant achievable rates on different channels and the demands from destinations, has been proposed to improve the spectrum efficiency and maximize the system throughput. Suppose the time frame of data transmission from SAP $v_{0}$ to the destination node $v_{j}$ via $\mathrm{RN} v_{i}$ is denoted by $T_{s}$, which is divided into two time slots $t_{0 i}$ and $t_{i j}$ for receiving and transmitting on two relay links in Figure 2, where $r_{j i}=$ 1. $\alpha_{j i}\left(0<\alpha_{j i}<1\right)$ denotes the DyTSA ratio for relay link from $v_{0}$ to $v_{j}$ via RN $v_{i}$, where $\alpha_{j i}=t_{0 i} / T_{s}$ and $T_{s}=$ $t_{0 i}+t_{i j}$. Also, $D=\left\{d_{i}\right\}$ denotes the transmission demand of $v_{i}$, where $d_{i} \geq 0, \forall i$. The throughput of $v_{i}$ is denoted by $\theta i$, which can be calculated in three scenarios below.

\subsection{Scenario 1}

Destination node $v_{i}$ has no relay link and is not acting as a $\mathrm{RN}$ either, which only receives data from $v_{0}$ via

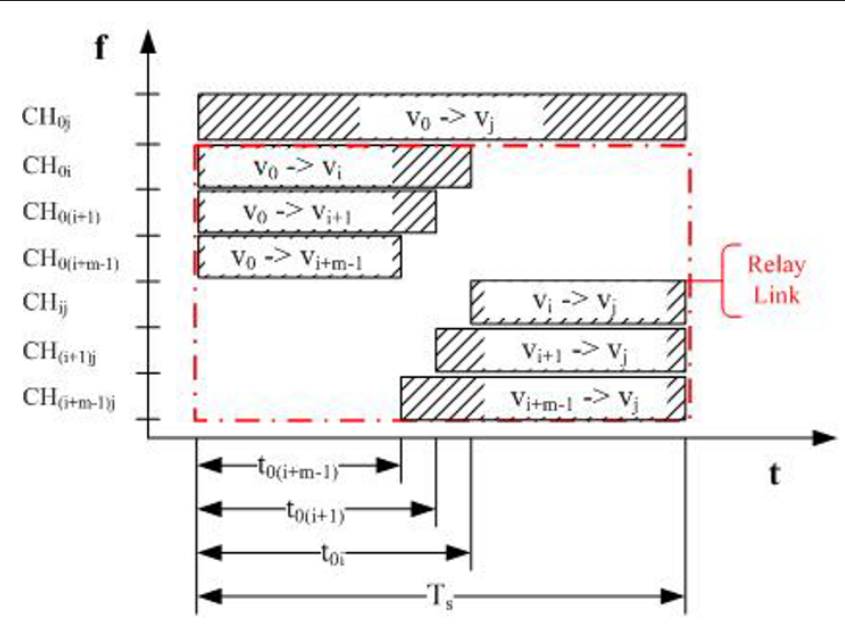

Figure 2 Flow of DyTSA scheme in MR-MD scenario. 
direct link with $r_{i j}=0$ and $r_{j i}=0, \forall j$. The throughput of $v_{i}$ is depicted by $\tilde{\theta}_{i}$ in (2), where $C_{0 i}=\sum_{k=1}^{K} c_{0 i}^{k} x_{0 i}^{k}$ is the sum of achievable rate between $v_{0}$ and $v_{i}$ and $d_{i}$ is its demand.

$$
\tilde{\theta}_{i}=\min \left(C_{0 i}, d_{i}\right)
$$

\subsection{Scenario 2}

Node $v_{i}$ acts as the RN between $v_{0}$ and $v_{j}$ with constraint that its demand is smaller than its achievable rate as $d_{i}<C_{0 i}$, where $r_{j i}=1$ and $r_{i j}=0$. The throughputs of its own data and relay data are depicted by $\bar{\theta}_{j i}$ and $\bar{\theta}_{j i}^{R}$ in (3).

$$
\left\{\begin{array}{l}
\bar{\theta}_{j i}=d_{i} \\
\bar{\theta}_{j i}^{R}=\alpha_{j i} C_{0 i}-d_{i}
\end{array}\right.
$$

\subsection{Scenario 3}

Node $v_{i}$ acts as the destination node with multiple RNs $v_{i}(1 \leq j \leq N, j \neq i)$, and the throughput of $v_{i}$ via $v_{j}$ is depicted by $\widehat{\theta}_{i j}^{R}$, where $r_{i j}=1$ and $r_{j i}=0$. Besides, the throughput of the direct link from $v_{0}$ to $v_{i}$ is depicted by $\widehat{\theta}_{i j}^{D}$ and the total throughput at node $v_{i}$ is depicted by $\widehat{\theta}_{i j}$ in (4), where $C_{j i}=\sum_{k=1}^{K} c_{j i}^{k} x_{j i}^{k}$.

$$
\left\{\begin{array}{l}
\hat{\theta}_{i j}^{R}=\left(1-\alpha_{i j}\right) C_{j i} \\
\hat{\theta}_{i j}^{D}=C_{0 i} \\
\widehat{\theta}_{i j}=\widehat{\theta}_{i j}^{R}+\widehat{\theta}_{i j}^{D}
\end{array}\right.
$$

In summary, the total throughput $\theta_{i}$ for node $v_{i}$ can be calculated by (5) in [18].

$$
\theta_{i}=\left(1-\sum_{j=1}^{N} r_{i j}\right)\left(1-\sum_{j=1}^{N} r_{j i}\right) \tilde{\theta}_{i}+\sum_{j=1}^{N} r_{j i} \bar{\theta}_{j i}+\sum_{j=1}^{N} r_{i j} \widehat{\theta}_{i j}
$$

\section{Propositions and proofs}

Considering the MR-MD scenario, five propositions and proofs are analyzed and proved below in detail, including the calculation of DyTSA ratio $\alpha_{j i}$ and the system throughput improvement of DyTSA scheme.

\subsection{Proposition 1}

Assume the RN $v_{i}$ serves $n$ destinations $\left\{v_{j}, v_{j+1}, \ldots, v_{j+n-1}\right\}$. $D=\left\{d_{i}\right\}$ depicts the demand of node $v_{i}$ and $C_{i j}=\sum_{k=1}^{K} c_{i j}^{k} x_{i j}^{k}$ depicts the sum of the achievable rate between $v_{i}$ and $v_{j}$. Due to the assumptions that the RN could not receive and transmit simultaneously, the optimal ratio from $v_{i}$ to its multiple destinations is depicted by $\alpha_{i}$ in (6), where $\alpha_{i}=\alpha_{q i}=t_{0 i} / T_{s}, q \in\{j, j+$ $1, \ldots, j+n-1\}$.

$$
\alpha_{i}=\alpha_{q i}=\frac{\left(d_{i}+\sum_{q=j}^{j+n-1} C_{i q}\right)}{\left(C_{0 i}+\sum_{q=j}^{j+n-1} C_{i q}\right)}
$$

Proof: For each relay link $r_{q i}=1$, where $q \in\{j, j+1, \ldots, j+$ $n-1\}$, the data transmitted from $v_{0}$ to $v_{i}$ must equal to the total data received at destinations $\left\{v_{j}, v_{j+1}, \ldots, v_{j+n-1}\right\}$ to maximize the system throughput as $\bar{\theta}_{i}^{R}=\sum_{q=j}^{j+n-1} \widehat{\theta}_{q i}^{R}$. Based on the formulas in (3)-(4) where $\bar{\theta}_{i}^{R}=\alpha_{i} C_{0 i}-d_{i}$ and $\hat{\theta}_{q i}^{R}=\left(1-\alpha_{q i}\right) C_{i q}$, ratio $\alpha_{i}$ is verified by (7), where $C_{i q}=\sum_{k=1}^{K} c_{i q}^{k} x_{i q}^{k} \geq 0, \quad C_{i q}=\sum_{k=1}^{K} c_{i q}^{k} x_{i q}^{k} \geq 0 \quad$ and $\because \bar{\theta}_{i}^{R}=\sum_{q=j}^{j+n-1} \widehat{\theta}_{q i}^{R}$

$\because \bar{\theta}_{i}^{R}=\alpha_{i} C_{0 i}-d_{i}$

$\because \sum_{q=j}^{j+n-1} \widehat{\theta}_{q i}^{R}=\sum_{q=j}^{j+n-1}\left(1-\alpha_{q i}\right) C_{i q}=\left(1-\alpha_{i}\right) \sum_{q=j}^{j+n-1} C_{i q}$.
$\therefore \alpha_{i} C_{0 i}-d_{i}=\left(1-\alpha_{i}\right) \sum_{q=j}^{j+n-1} C_{i q}$

$\therefore \alpha_{i}=\frac{\left(d_{i}+\sum_{q=j}^{j+n-1} C_{i q}\right)}{\left(C_{0 i}+\sum_{q=j}^{j+n-1} C_{i q}\right)}$

$\because \bar{\theta}_{i}^{R}=\sum_{q=j}^{j+n-1} \widehat{\theta}_{q i}^{R}$

$\because \bar{\theta}_{i}^{R}=\alpha_{i} C_{0 i}-d_{i}$

$\because \sum_{q=j}^{j+n-1} \widehat{\theta}_{q i}^{R}=\sum_{q=j}^{j+n-1}\left(1-\alpha_{q i}\right) C_{i q}=\left(1-\alpha_{i}\right) \sum_{q=j}^{j+n-1} C_{i q}$

$\therefore \alpha_{i} C_{0 i}-d_{i}=\left(1-\alpha_{i}\right) \sum_{q=j}^{j+n-1} C_{i q}$

$\therefore \alpha_{i}=\frac{\left(d_{i}+\sum_{q=j}^{j+n-1} C_{i q}\right)}{\left(C_{0 i}+\sum_{q=j}^{j+n-1} C_{i q}\right)}$

\subsection{Proposition 2}

For $\mathrm{RN} v_{i}$ selection, it must confine to the condition that its demand is no bigger than its achievable rate in (8).

$$
0 \leq d_{i} \leq C_{0 i}
$$


Proof: Chosen as the $\mathrm{RN} v_{i}$, its achievable rate $C_{0 i}$ will change to $\alpha{ }_{i} C_{0 i}$ based on the DyTSA scheme, which should be no smaller than its demand $d_{i}$ as $\alpha{ }_{i} C_{0 i} \geq d_{i}$ to fulfill the relay task to multiple destinations as shown in (9), where $C_{0 i} \geq 0, \sum_{q=j}^{j+n-1} C_{i q}>0$ and $C_{0 i}+\sum_{q=j}^{j+n-1} C_{i q}>0$.

$$
\begin{aligned}
& \because \alpha_{i} C_{0 i} \geq d_{i} \\
& \therefore \alpha_{i} C_{0 i}-d_{i}=\frac{\left(C_{0 i}-d_{i}\right) \sum_{q=j}^{j+n-1} C_{i q}}{\left(C_{0 i}+\sum_{q=j}^{j+n-1} C_{i q}\right)} \geq 0 \\
& \therefore 0 \leq d_{i} \leq C_{0 i}
\end{aligned}
$$

\subsection{Proposition 3}

The throughput of dynamic scheme for relay link is no smaller than that of the fixed scheme in [18] by (10), where

$$
\begin{aligned}
& \sum_{q-j}^{j+n-1} \bar{\theta}_{q r}^{R-f i x}=\min \left(C_{o r} / 2-d_{r}, \frac{1}{2} \sum_{q=j}^{j+n-1} C_{r q}\right), \sum_{q=j}^{j+n-1} \theta_{q r}^{R-f i x}=\min \left(C_{0 r} / 2-d_{r}, \frac{1}{2} \sum_{q q j}^{j+n-1} C_{r q}\right), \\
& C_{0 r} \geq 0, \sum_{q=j}^{j+n-1} C_{r q}>0, C_{0 r}+\sum_{q=j}^{j+n-1} C_{r q}>0,0 \leq d_{r} \leq C_{0 r} / 2 . \\
& \sum_{r=i}^{i+m-1} \bar{\theta}_{r}^{R} \geq \sum_{r=i}^{i+m-1} \sum_{q=j}^{j+n-1} \bar{\theta}_{q r}^{R-f i x}
\end{aligned}
$$

Proof: Let us define $\Delta=\sum_{r=i}^{i+m-1} \bar{\theta}_{r}^{R}-\sum_{r=i}^{i+m-1} \sum_{q=j}^{j+n-1} \bar{\theta}_{q r}^{R-f i x}$ and the validity of (10) transforms to prove $\Delta \geq 0$, which are proved by three cases below.

\subsubsection{Case 1}

When

$$
0 \leq C_{0 r} / 2-d_{r}<\frac{1}{2} \sum_{q=j}^{j+n-1} C_{r q},
$$

then

$$
\begin{aligned}
& C_{0 r}-\sum_{q=j}^{j+n-1} C_{r q}-2 d_{r}<0 \\
& \because \Delta_{1}=\sum_{r=i}^{i+m-1}\left[\left(1-\alpha_{r}\right) \sum_{q=j}^{j+n-1} C_{r q}\right]-\sum_{r=i}^{i+m-1}\left(\frac{C_{0 r}}{2}-d_{r}\right) . \\
& \because \Delta_{1}=\sum_{r=i}^{i+m-1}\left[\left(1-\alpha_{r}\right) \sum_{q=j}^{j+n-1} C_{r q}\right]-\sum_{r=i}^{i+m-1}\left(\frac{C_{0 r}}{2}-d_{r}\right)
\end{aligned}
$$

$$
\begin{aligned}
& =\sum_{r=i}^{i+m-1}\left(\frac{C_{0 r}-d_{r}}{C_{0 r}+\sum_{q=j}^{j+n-1} C_{r q}} \sum_{q=j}^{j+n-1} C_{r q}-\frac{C_{0 r}}{2}+d_{r}\right) \\
& =-\sum_{r=i}^{i+m-1} \frac{C_{0 r}\left(C_{0 r}-\sum_{q=j}^{j+n-1} C_{r q}-2 d_{r}\right)}{2\left(C_{0 r}+\sum_{q=j}^{j+n-1} C_{r q}\right)}>0 \\
& \therefore \Delta_{1}>0
\end{aligned}
$$

\subsubsection{Case 2}

When

$$
C_{0 r} / 2-d_{r}>\frac{1}{2} \sum_{q=j}^{j+n-1} C_{r q},
$$

then

$$
C_{0 r}-\sum_{q=j}^{j+n-1} C_{r q}-2 d_{r}>0
$$

and

$$
\begin{aligned}
\because \Delta_{2} & =\sum_{r=i}^{i+m-1}\left[\left(1-\alpha_{r}\right) \sum_{q=j}^{j+n-1} C_{r q}\right]-\sum_{r=i}^{i+m-1} \frac{1}{2} \sum_{q=j}^{j+n-1} C_{r q} . \\
\because & \Delta_{2}=\sum_{r=i}^{i+m-1}\left[\left(1-\alpha_{r}\right) \sum_{q=j}^{j+n-1} C_{r q}\right]-\sum_{r=i}^{i+m-1} \frac{1}{2} \sum_{q=j}^{j+n-1} C_{r q} \\
& =\sum_{r=i}^{i+m-1}\left(\frac{C_{0 r}-d_{r}}{C_{0 r}+\sum_{q=j}^{j+n-1} C_{r q}} \sum_{q=j}^{j+n-1} C_{r q}-\frac{1}{2} \sum_{q=j}^{j+n-1} C_{r q}\right) \\
& =\sum_{r=i}^{i+m-1} \frac{\left(C_{0 r}-\sum_{q=j}^{j+n-1} C_{r q}-2 d_{r}\right) \sum_{q=j}^{j+n-1} C_{r q}}{2\left(C_{0 r}+\sum_{q=j}^{j+n-1} C_{r q}\right)}>0 \\
\therefore & \Delta_{2}>0
\end{aligned}
$$

\subsubsection{Case 3}

When $\quad C_{0 r} / 2-d_{r}=\frac{1}{2} \sum_{q=j}^{j+n-1} C_{r q}$, then

$C_{0 r}-\sum_{q=j}^{j+n-1} C_{r q}-2 d_{r}=0 \quad$ and

$$
\begin{gathered}
\because \Delta_{3}=\sum_{r=i}^{i+m-1}\left[\left(1-\alpha_{r}\right) \sum_{q=j}^{j+n-1} C_{r q}\right]-\sum_{r=i}^{i+m-1} \frac{1}{2} \sum_{q=j}^{j+n-1} C_{r q} . \\
\because \Delta_{3}=\sum_{r=i}^{i+m-1}\left[\left(1-\alpha_{r}\right) \sum_{q=j}^{j+n-1} C_{r q}\right]-\sum_{r=i}^{i+m-1} \frac{1}{2} \sum_{q=j}^{j+n-1} C_{r q}
\end{gathered}
$$

$$
\begin{aligned}
& =\sum_{r=i}^{i+m-1} \frac{\left(C_{0 r}-\sum_{q=j}^{j+n-1} C_{r q}-2 d_{r}\right) \sum_{q=j}^{j+n-1} C_{r q}}{2\left(C_{0 r}+\sum_{q=j}^{j+n-1} C_{r q}\right)}=0 \\
& \therefore \Delta_{3}=0
\end{aligned}
$$

In summary, $\Delta \geq 0$ is correct based on the proofs in (13)-(20), which also proves the proposition in (10).

\subsection{Proposition 4}

The demand $d_{j}$ of destination node $v_{j}$ equals to the sum of data from both direct link and multiple relay links, where $d_{j}=C_{0 j}+\sum_{r=i}^{i+m-1} \widehat{\theta}_{j r}^{R}$. The demand of $v_{j}$ is depicted in (21).

$$
d_{j}=C_{0 j}+\sum_{r=i}^{i+m-1} \frac{\left(C_{0 r}-d_{r}\right) C_{r j}}{C_{0 r}+\sum_{q=j}^{j+n-1} C_{r q}}
$$


Proof: Under the assumption that the demand $d_{j}$ of destination node $v_{j}$ equals to the sum of data from both direct link and multiple relay links, $d_{j}$ is verified in (25), where $\hat{\theta}_{j r}^{R}=\left(1-\alpha_{j r}\right) C_{r j}$

$$
\begin{aligned}
& \because d_{j}=C_{0 j}+\sum_{r=l}^{i+m-1} \widehat{\theta}_{j r}^{R} . \\
& \because d_{j}=C_{0 j}+\sum_{r=l}^{i+m-1} \widehat{\theta}_{j r}^{R} \\
& \therefore d_{j}=C_{0 j}+\sum_{r=i}^{i+m-1}\left[\left(1-\alpha_{j r}\right) C_{r j}\right] \\
& \therefore d_{j}=C_{0 j}+\sum_{r=i}^{i+m-1}\left[\left(1-\frac{d_{r}+\sum_{q=j}^{j+n-1} C_{r q}}{C_{0 r}+\sum_{q=j}^{j+n-1} C_{r q}}\right) C_{r j}\right] \\
& \therefore d_{j}=C_{0 j}+\sum_{r=i}^{i+m-1} \frac{\left(C_{0 r}-d_{r}\right) C_{r j}}{C_{0 r}+\sum_{q=j}^{j+n-1} C_{r q}}
\end{aligned}
$$

\subsection{Proposition 5}

The calculation formula of the throughput $\theta_{i}$ for node $v_{i}$, which is applicable in the scenario of one RN serving one destination node in [18], is also applicable in the MR-MD scenario as shown in (5).

Proof: Under the scenario of MR-MD nodes, the sum of the relay links for destination node $v_{i}$ via $\mathrm{RN} v_{j}$ is larger than 1 , where $\sum_{j=1}^{N} r_{i j}>1$. For destination node $v_{i}$, its throughput is calculated as in (5), which is proved by (29) based on (2) and (4), where $\sum_{j=1}^{N} r_{i j}>1, \sum_{j=1}^{N} r_{j i}=0$ and $d_{i}>C_{0 i}$. Finally, the throughput of $v_{i}$ equals to its demand $d_{i}$ via relay links.

$$
\begin{aligned}
& \therefore \theta_{i}=\left(1-\sum_{j=1}^{N} r_{i j}\right)\left(1-\sum_{j=1}^{N} r_{j i}\right) \tilde{\theta}_{i}+\sum_{j=1}^{N} r_{j i} \bar{\theta}_{j i}+\sum_{j=1}^{N} r_{i j} \hat{\theta}_{i j} \\
& \therefore \theta_{i}=\left(1-\sum_{j=1}^{N} r_{i j}\right) \tilde{\theta}_{i}+\sum_{j=1}^{N} r_{i j} \widehat{\theta}_{i j} \\
& \therefore \theta_{i}=\left(1-\sum_{j=1}^{N} r_{i j}\right) C_{0 i}+\sum_{j=1}^{N} r_{i j}\left(\widehat{\theta}_{i j}^{R}+C_{0 i}\right) \\
& \therefore \theta_{i}=C_{0 i}+\sum_{j=1}^{N} r_{i j} \widehat{\theta}_{i j}^{R}=d_{i}
\end{aligned}
$$

\section{Joint RN selection, channel allocation and DyTSA scheme}

Based on the theoretical model and analysis of the throughput $\theta_{i}$ for node $v_{i}$ in (5), the total throughput of all nodes in CRNs is depicted by $\sum_{i=1}^{N} \theta_{i}$ in (30). Considering the MR-MD scenario in CRNs, the constraints in $[17,18]$, where $\quad \sum_{j=1}^{N} r_{i j} \leq 1,1 \leq i \leq N \quad$ and $\sum_{i=1}^{N} r_{i j} \leq 1,1 \leq j \leq N$, will not be satisfied by all nodes. So by appropriately applying the joint RN selection, channel allocation and DyTSA scheme $(R, X, \alpha)$, the maximal total system throughput can be achieved by using the max fiow theory [19] as shown in (35).

$$
\begin{aligned}
& \sum_{i=1}^{N} \theta_{i}=\sum_{i=1}^{N}\left[\left(1-\sum_{j=1}^{N} r_{i j}\right)\left(1-\sum_{j=1}^{N} r_{j i}\right) \tilde{\theta}_{i}+\sum_{j=1}^{N} r_{j i} \bar{\theta}_{j i}+\sum_{j=1}^{N} r_{i j} \hat{\theta}_{i j}\right] \\
& \max _{R, X, \alpha} \sum_{i=1}^{N} \theta_{i}
\end{aligned}
$$

$$
\begin{aligned}
& \text { s.t. } r_{i j} \sum_{j^{\prime}=1}^{N} r_{j^{\prime} i}=0,1 \leq i \leq N, \quad 1 \leq j \leq N \\
& r_{i j} \leq e_{i j}, 1 \leq i \leq N, 1 \leq j \leq N
\end{aligned}
$$

$$
\sum_{e_{i j} \in E}^{N} x_{i j}^{k} \leq 1, \forall k
$$

$$
x_{i j}^{k} \leq a_{i}^{k} a_{j}^{k}, \forall i, j, k
$$

The process of proposed joint RN selection, channel allocation and DyTSA scheme takes six steps as shown in Figure 3 and is depicted below in detail.

Step 1: Allocate available channels to direct links from SAP node $v_{0}$ to all SU nodes $v_{i},(1<i<N)$ in the centralized cooperative scenario.

Step 2: Divide nodes into two categories: spectrum "rich" nodes $\left(0 \leq d_{i} \leq C_{0 i}\right)$ as relay and "starving" nodes $\left(d_{i}>C_{0 i}\right)$ as destination.

Step 3: Sort all "starving" destination nodes based on its resource starving intensity as depicted by $\left(d_{i}-C_{0 i}\right)$ in descending order which is denoted by the set $D=\left\{v_{i} \mid d_{i}>C_{0 i}\right.$, 


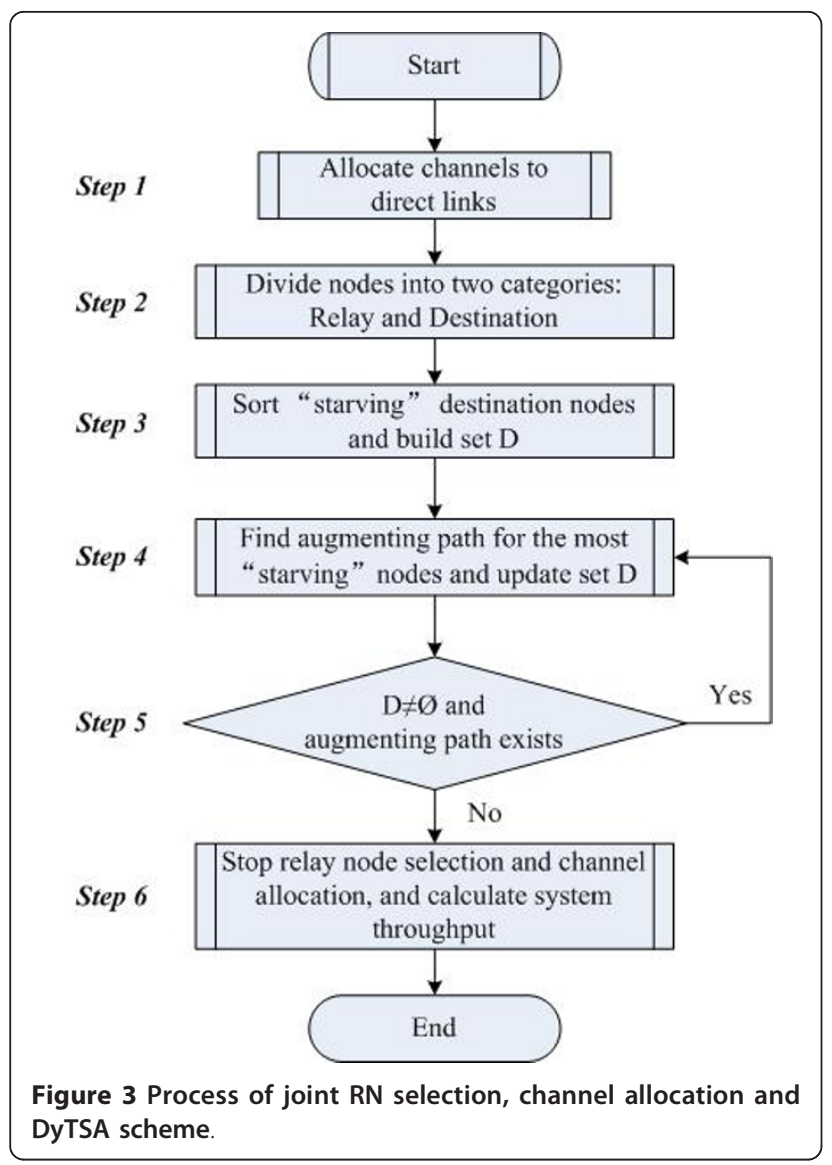

$\left.1 \leq i \leq N_{\text {strvv }}\right\}$, where $N_{\text {strv }}$ is the total number of starving nodes and $\left(1<N_{\text {strv }}<N\right)$.

Step 4: First, select the most "starving" node $D_{j}$ from set $\mathrm{D}$ as the destination node which need RNs, where $D_{j}=\left\{j \mid j=\operatorname{argmax} d j>C_{0 j}, 1 \leq j \leq N_{\text {strv }}\right\}$. Second, apply the augmenting path algorithm for max fiow to select appropriate $\mathrm{RN} v_{i}$ for destination node $D_{j}$ and allocate channel for relay data transmission for $D_{j}$. Third, based on the DyTSA scheme design optimal DyTSA ratio $\alpha_{j i}$ between RN $v_{i}$ and destination node $D_{j}$. And the fiow of original RN $v_{i}$ is depicted by $f_{0 i}$ where $f_{0 i}=d_{i}$. Therefore, the relay data from $v_{i}$ to $D_{j}$ via path $P_{j}$ is denoted by $\tau\left(P_{j}\right)$, where $\tau\left(P_{j}\right)=\alpha_{j i} C_{0 i}-f_{0 i}$. By applying the augmenting path algorithm, the fiow for $D_{j}$ has been changed to $f_{i}^{\prime}$, where $f_{i}^{\prime}=f_{i}+\tau\left(P_{j}\right)$. Finally, delete node $D v$ from the set $D$ and update the order in "starving" destination set $D$.

Step 5: Check whether the set $D$ is empty. If the "starving" destination set $D \neq \varphi$ and augmenting path exists, then go back to Step 4 to find the possible augmenting path for the existing "starving" nodes. Otherwise, if $D=\varphi$ or no possible augmenting path could be found, it means that all nodes' demands are satisfied or no more spectrum and link resources are available in CRNs, then go to Step 6.
Step 6: Stop the process and calculate the system throughput of all nodes $\sum_{i=1}^{N} \theta_{i}$.

\section{Simulation results and analysis}

\subsection{Simulation setup}

Considering the MR-MD scenario in CRNs as shown in Figure 4, parameters for simulation are shown in Table 1 which are based on 3GPP Case 1 relay scenario [20]. The signal to noise ratio (SNR) of RN and SU are depicted in (36)-(37). Suppose each user occupies only one resource block (RB) and the achievable capacity of each RB in cooperative relay network is depicted by (38).

$$
\begin{aligned}
& \operatorname{SNR}_{\mathrm{RN}}\left(R_{0 i}\right)=P_{\mathrm{SAP}}-\mathrm{PL}_{\mathrm{RN}}\left(R_{0 i}\right)-\mathrm{NP} \\
& \operatorname{SNR}_{\mathrm{SU}}\left(R_{i j}\right)=P_{\mathrm{RN}}-\mathrm{PL}_{\mathrm{SU}}\left(R_{i j}\right)-\mathrm{NP} \\
& c_{i j}=\log _{2}\left[1+\operatorname{SNR}\left(R_{i j}\right)\right]
\end{aligned}
$$

\subsection{Results analysis}

Based on the parameters in Table 1, the SUs are randomly deployed in the simulation region with SAP radius $100 \mathrm{~m}$. The capacity of CRNs by using DyTSA and fix relay schemes are simulated and analyzed with different SU density. As shown in Figures 5, 6, and 7, respectively, by dynamically tuning the DyTSA ratio $\alpha_{j i}$ to maximize the relay data transmission with different capacity on receiving and transmitting links, the maximum system throughput of CRNs can be achieved by applying the DyTSA scheme in contrast to the fixed scheme. Moreover, as the RNs increase, the vacant

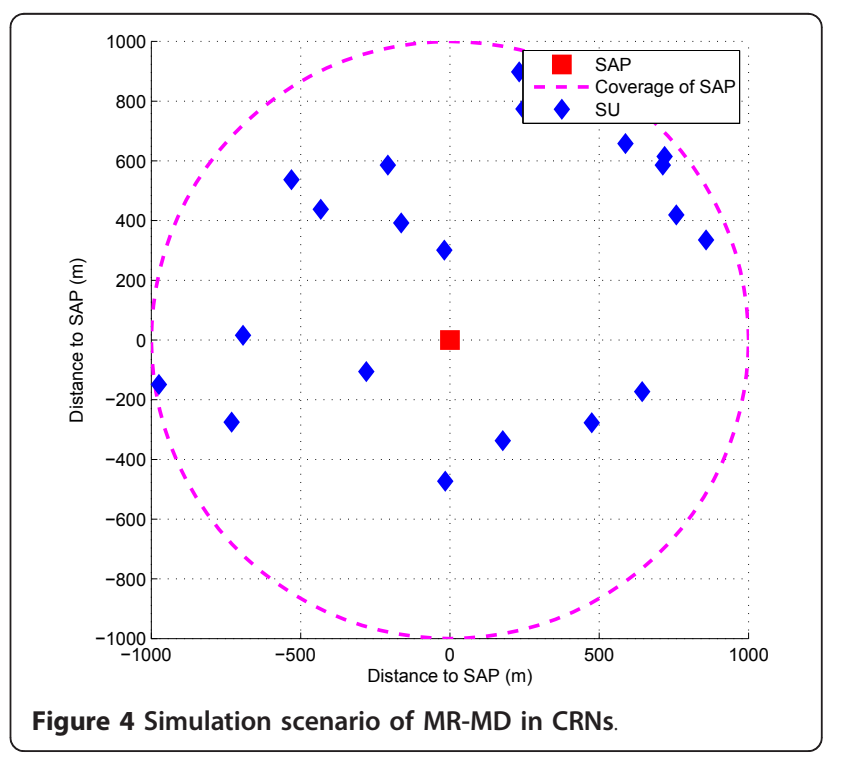


Table 1 Parameters for cooperative relay in CRNs

\begin{tabular}{ll}
\hline$P_{\mathrm{SAP}}(\mathrm{dBm})$ & 30 \\
$P_{\mathrm{RN}}(\mathrm{dBm})$ & 23 \\
System bandwidth BW(MHz) & 10 \\
Number of resource block (RB) & 50 \\
Bandwidth of each RB BW $\mathrm{RB}(\mathrm{MHz})$ & 0.2 \\
Path loss model from SAP to RN $(R: \mathrm{km})$ & $\mathrm{PL}_{\mathrm{RN}}(R)=100.7+$ \\
& $23.5 \log _{10} R$ \\
& $\mathrm{PL}_{\mathrm{SU}}(R)=103.8+$ \\
Path loss model from RN to SU $(R: \mathrm{km})$ & $20.9 \log _{10} R$ \\
& $10 \log _{10}(\mathrm{kTNFBW})$ \\
Received noise power NP $(\mathrm{dBm})$ & $1.3804 \times 10^{-20} \times 290$ \\
$\mathrm{kT}$ (mW/Hz) & 5 \\
$\mathrm{NF}(\mathrm{dB})$ & 1,732 \\
Distance between inter base station ISD & \\
(m) & $10,30,50$ \\
Number of SU N $\mathrm{sU}$ & 100 \\
Simulation time/sample & \\
\hline
\end{tabular}

spectrum can be utilized much more efficiently, which greatly improve the system capacity in CRNs.

Furthermore, the system capacity improvement by using DyTSA scheme is analyzed and compared to the fixed scheme with the increase of DyTSA ratio $\alpha_{j i}$ as shown in Figure 8. Results shown that the system capacity of DyTSA scheme is no smaller than that of fixed scheme and the equilibrium point of two schemes is $\alpha_{j i}$ $=0.5$. Apart from the equilibrium point, $\alpha_{j i}=0.5$, the system capacity of DyTSA scheme is always bigger than that of fixed scheme as $\alpha_{j i}$ increases, where $0<\alpha_{j i}<1$. Two regions of system capacity improvement are highlighted in Figure 8. Moreover, the fixed scheme is only effective when $0 \leq d_{i} \leq C_{0 i} / 2$, while the DyTSA scheme is applicable in the general cases when $0 \leq d_{i} \leq C_{0 i}$, which is considered as a great improvement to the existing research works.

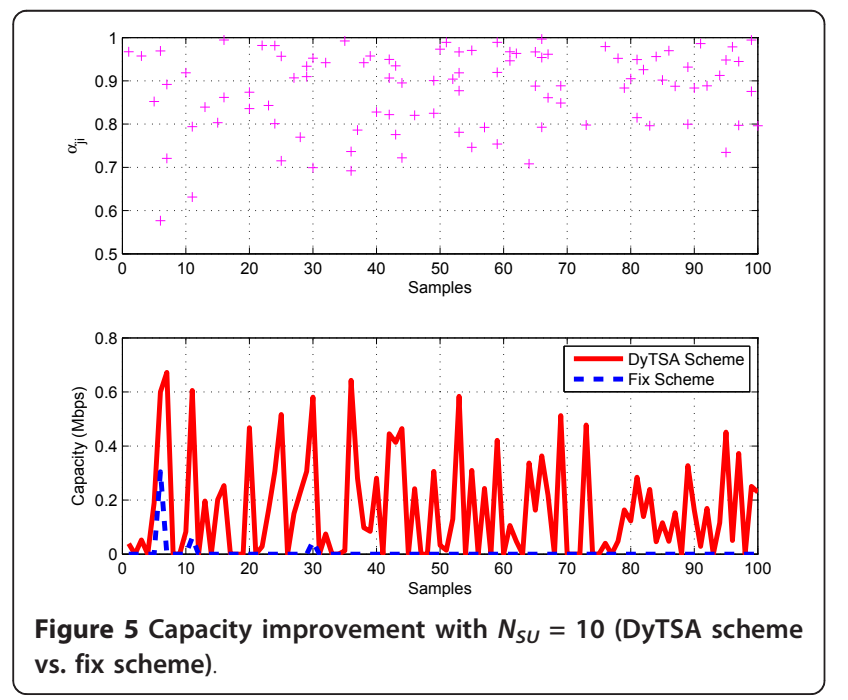

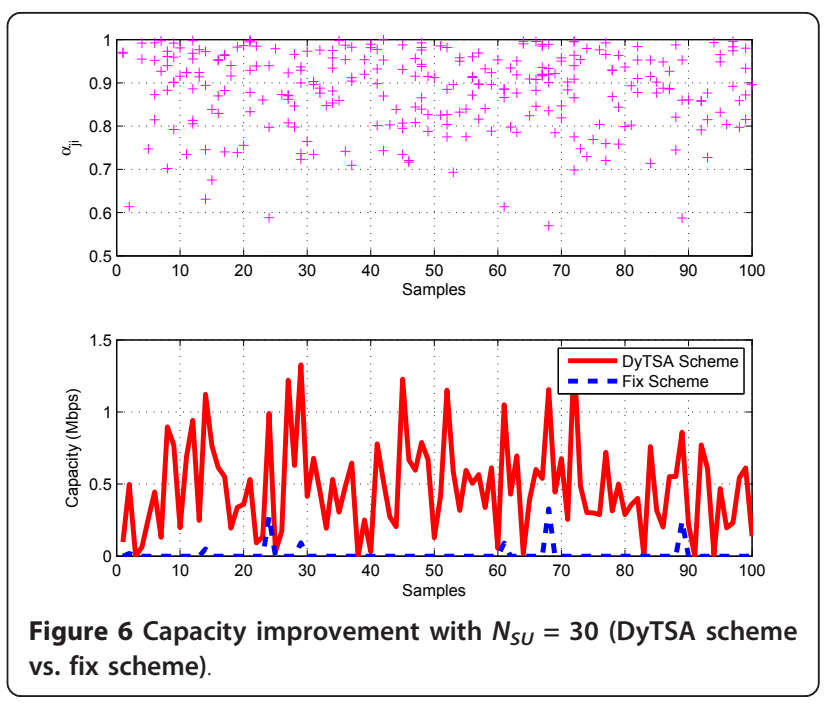

\section{Conclusion and future work}

As a novel solution to improve the spectrum efficiency and system capacity in CRNs, the DyTSA based joint cooperative relay optimal scheme has been proposed and proved theoretically in this article. By dynamically tuning the DyTSA ratio on different relay links, the system capacity has increased tremendously comparing to the traditional fixed scheme. Moreover, by applying the joint RN selection, channel allocation and DyTSA scheme in MR-MD scenario, both the spectrum efficiency and system capacity have been improved greatly and verified by numerous results.

Further studies on multi-hop cooperative relay schemes in CRNs need pay much attention on interference constraints from neighbor PUs and appropriate power control scheme for RNs.

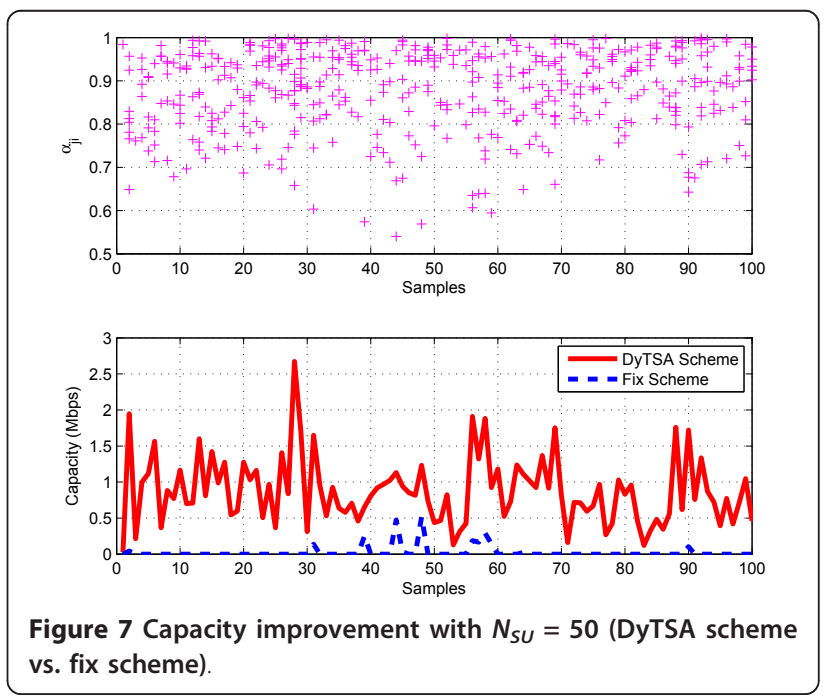




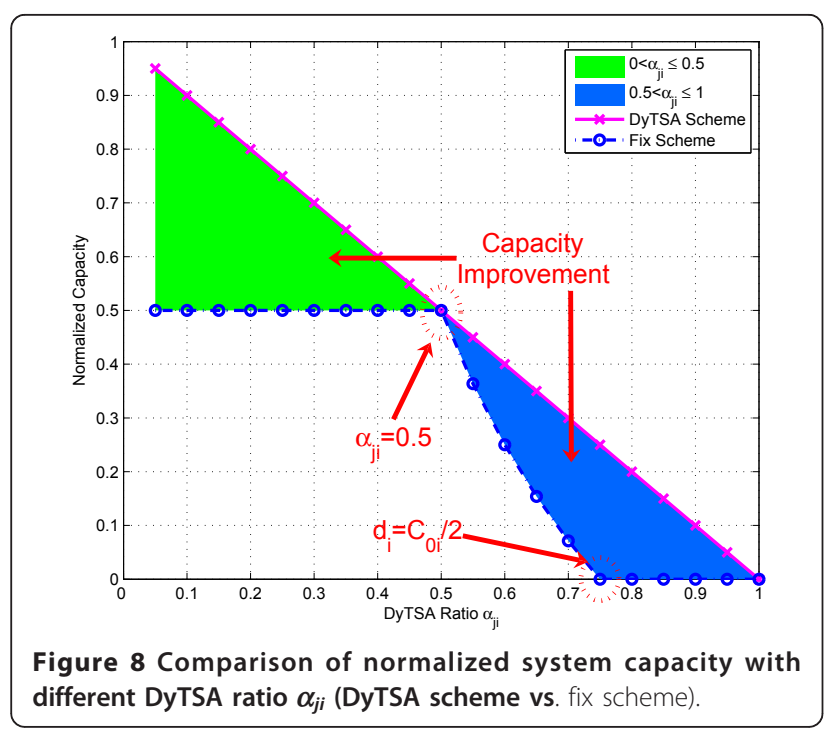

\section{Acknowledgements}

The authors would like to thank the colleagues from the Wireless Technology Innovation Institute of BUPT. This work was sponsored by the National Basic Research Program of China (2009CB320400), National Key Technology R\&D Program of China (2010ZX03003-001-01), National Natural Science Foundation of China $(60832009,61121001)$ and Program for New Century Excellent Talents in University (NCET-01-0259).

\section{Competing interests}

The authors declare that they have no competing interests.

Received: 30 June 2011 Accepted: 8 February 2012

Published: 8 February 2012

\section{References}

1. FCC, Spectrum Policy Task Force, Rep. ET Docket No. 02-135. (2002)

2. FCC, Facilitating opportunities for flexible, efficient and reliable spectrum use employing cognitive radio technologies: notice of proposed rule making and order, FCC Document ET Docket No. 03-108. (2003)

3. J Mitola, Cognitive radio, Licentiate proposal, KTH, Stockholm, Sweden. (1998)

4. J Mitola, Software Radios: Wireless Architecture for the 21st Century, (Wiley, New York, 2000)

5. P Demestichas, G Dimitrakopoulos, J Strassner, D Bourse, Introducing reconfigurability and cognitive networks concepts in the wireless world. IEEE Veh Technol Mag. 1(2), 32-39 (2006)

6. KB Letaief, W Zhang, Cooperative communications for cognitive radio networks. Proc IEEE. 97(5), 878-893 (2009)

7. Y Guo, G Kang, N Zhang, W Zhou, P Zhang, Outage performance of relayassisted cognitive-radio system under spectrum-sharing constraints. Electron Lett. 46(2), 182-184 (2010). doi:10.1049/el.2010.2159

8. O Simeone, Y Bar-Ness, U Spagnolini, Stable throughput of cognitive radios with and without relaying capability. IEEE Trans. Commun. 55(12), 2351-2360 (2007)

9. AK Sadek, Z Han, KJR Liu, A distributed relay-assignment algorithm for cooperative communications in wireless networks, in Proc IEEE International Conference on Communications ICC'O6, vol. 4. Istanbul, Turkey, 1592-1597 (July 2006)

10. AK Sadek, Z Han, KIR Liu, Distributed relay-assignment protocols for coverage expansion in cooperative wireless networks. IEEE Trans Mobile Comput. 9(4), 505-515 (2010)

11. B Wang, Z Han, KIR Liu, Distributed relay selection and power control for multiuser cooperative communication networks using buyer/seller game, in Proc IEEE INFOCOM, Anchorage, Alaska, 544-552 (2007)

12. S Sharma, Y Shi, YT Hou, HD Sherali, S Kompella, Cooperative Communications in multi-hop wireless networks: joint fiow routing and relay node assignment, in IEEE INFOCOM 2010, San Diego, CA, USA, 1-9 (May 2010)

13. Y Shi, S Sharma, YT Hou, HD Sherali, S Kompella, SF Midkiff, Optimal relay assignment for cooperative communications, in Proc of ACM MobiHoc, Hongkong, China, 3-12 (2008)

14. M Xie, W Zhang, K Wong, A geometic approach to improve spectrum efficiency for cognitive relay networks. IEEE Trans Wirel Commun. 9(1), 268-281 (2010)

15. TC-Y Ng, W Yu, Joint optimization of relay strategies and resource allocations in cooperative cellular networks. IEEE J Sel Areas Commun. 25(2), 328-339 (2007)

16. EM Yeh, RA Berry, Throughput optimal control of cooperative relay networks. IEEE Trans Inf Theory 53(10), 3827-3833 (2007)

17. Q Zhang, J Jia, J Zhang, Cooperative relay to improve diversity in cognitive radio networks. IEEE Commun Mag. 47(2), 111-117 (2009)

18. J Jia, J Zhang, Q Zhang, Cooperative Relay for cognitive radio networks, in IEEE INFOCOM 2009, Rio de Janeiro, Brazil, pp. 2304-2312 (19-25 April 2009)

19. R Diestel, Graph Theory, 3rd edn. (Springer, Heidelberg, 2005)

20. 3GPP TR 36.814 V9.0.0 3GPP TSG RAN (E-UTRA), Further advancements for E-UTRA physical layer aspects (Release 9). (2010)

doi:10.1186/1687-1499-2012-37

Cite this article as: Zhang et al:: Joint cooperative relay scheme for spectrum-efficient usage and capacity improvement in cognitive radio networks. EURASIP Journal on Wireless Communications and Networking 2012 2012:37.

\section{Submit your manuscript to a SpringerOpen ${ }^{\mathcal{O}}$ journal and benefit from:}

- Convenient online submission

- Rigorous peer review

- Immediate publication on acceptance

- Open access: articles freely available online

- High visibility within the field

- Retaining the copyright to your article

Submit your next manuscript at $\gg$ springeropen.com 